\title{
Detailed structural elucidation of polyesters and acrylates using Fourier transform mass spectrometry
}

\author{
William J. Simonsick Jr. • Violeta I. Petkovska
}

Received: 15 May 2008 / Revised: 25 July 2008 / Accepted: 25 July 2008 / Published online: 22 August 2008

(C) Springer-Verlag 2008

\begin{abstract}
The detailed structural characterization of complex polymer architectures, like copolymers and polymer mixtures, by mass spectrometry presents a challenge. Even though soft ionization analyses revolutionized the characterization of large molecules and provided a means for determining the polymer's molecular weight distribution, polydispersity, and end groups, full microstructure elucidation and monomer sequencing by soft ionization alone is not possible. The combination of high-resolution Fourier transform mass spectrometry (FTMS) and tandem mass spectrometry $\left(\mathrm{MS}^{\mathrm{n}}\right)$ provides a powerful analytical tool for addressing these challenges. This tool was used in our work to separate and identify the products of polymerization between 12-hydroxystearic acid (HSA) and stearic acid (SA), to provide precise information about the exact location of caprolactones on the Tris(2-hydroxyethyl)isocyanurate (THEIC) molecule, and to sequence a glycidyl methacrylate/methyl methacrylate (GMA/MMA) copolymer. The results highlight the value of ultrahigh resolution and tandem mass spectrometry for fine structural characterization and sequencing of polymers.
\end{abstract}

Keywords Electrospray ionization $\cdot$ Laser desorption ionization - Fourier transform mass spectrometry . Collision-induced dissociation · Polymers · Copolymers . Tandem mass spectrometry

W. J. Simonsick Jr. ( $\bowtie)$

Chestnut Run Plaza, DuPont Fluorochemicals, Wilmington, DE 19880-0711, USA

e-mail: William.J.Simonsick@usa.dupont.com

V. I. Petkovska

Marshall R\&D Laboratory, DuPont Performance Coatings, Philadelphia, PA 19146, USA

\section{Introduction}

In order to comply with government regulations on organic solvent emission, the reduction of the volatile organic content (VOC) via high-solids formulations has become one of the most important approaches in the automotive coatings industry [1]. To achieve low VOC, the formulations are designed with highly functionalized low molecular weight polymers and crosslinkers, which allows the formation of crosslinked networks. These building blocks or precursors to crosslinked networks require less solvent to maintain sprayable viscosities and therefore provide highsolids, low-VOC formulations that yield long-lasting, durable films. The end-use properties of the crosslinked networks are strongly dependent on the precursors or building blocks. Furthermore, structural imperfections in the organic molecules used in high-solids formulations have a more profound impact on the final properties than imperfections in traditional low-solids high molecular weight compositions. As a result, analytical techniques that provide detailed information about the exact molecular contents of today's high-solids formulations have become necessary tools for characterization.

With the development of soft ionization techniques, mass spectrometry has emerged as a valuable tool for polymer characterization. Since the mass range of modern coatings components is amenable to the capabilities of commercial mass spectrometers, soft ionization techniques such as electrospray ionization (ESI) [2-6] and laser desorption (LD) [7, 8] are routinely used for coatings characterization. Not only do these techniques provide information about the polydispersity and the molecular weight distribution of polymers, but they also give information about the molecules' structural and elemental composition, such as repeat unit and end groups [9]. 
Despite its high value, however, single-stage mass spectrometry cannot provide information about the sequences and sometimes the accurate structures of complex polymer compositions like copolymers, for example. Since copolymer networks are the dominant architectures in coating formulations, their full structural characterization by single-stage mass spectrometry is a challenge. Two of the most common solutions to this shortcoming are to use high-resolution Fourier transform (FT) mass spectrometry and/or tandem mass spectrometry $\left(M^{n}\right)$. However, the high mass accuracy offered by high-resolution FTMS does not offer a means for monomer sequencing. In this case, multistage mass spectrometry experiments become critical for obtaining detailed structural and sequence information for the oligomeric copolymer.

Over the years, tandem mass spectrometry has been used with or without high resolution for polymer characterization. In 1997, Jackson et al. applied matrix-assisted laser desorption/ionization collision-induced dissociation (MALDICID) MS/MS to confirm the end-group compositions of polystyrene (PS) and poly(methyl methacrylate) (PMMA) standards $[10,11]$. Tandem mass spectrometry was also used to positively identify the major components of ethylene oxide/propylene oxide (EO/PO) copolymers [12] and to derive the fragmentation mechanism of polyglycols $[13,14]$ and poly(butylene adipate) [15]. The variety of polymer molecules studied by $\mathrm{MS}^{\mathrm{n}}$ extends to poly(ethylene glycol)s (PEGs) and poly ( $\alpha$-methyl styrene) [16], poly(butyl methacrylate) (PBMA) [17], nonpolar hydrocarbons such as polyisoprene [18], etc. For copolymer analyses, tandem mass spectrometry has proven to be invaluable in determinations of the block length, end-group structure, and copolymer sequence, as shown in the MS/MS characterization of polyester anions and cations of isophthalic acid and neopentyl glycol [19].

In this work, we show the capabilities of high-resolution FT alone and combined with MS/MS for the characterization of modern coating components. Fourier transform mass spectrometry has become a very useful analytical tool due to its high mass accuracy, high sensitivity, and ultrahigh mass resolving power over a broad $m / z$ range [18]. To achieve ultrahigh resolution for the purpose of this investigation, the method of choice was quadrupolar excitation activation (QEA). This technique was first introduced and described in detail by Marshall and coworkers [20] and later applied to polymers by Wilkins [18]. In brief, during the QEA experiments, the undesirable magnetron motion of the ions is converted to cyclotron motion. The ions are collisionally relaxed to the center of the cell, transferred to a lower pressure region, excited, and detected, affording ultrahigh resolution [21]

A commonly used activation method in FTMS is sustained off-resonance irradiation collision-induced dissocia- tion (SORI-CID) $[22,23]$. During the SORI-CID process, ions are excited by applying a radiofrequency at about $1000 \mathrm{~Hz}$ below or above their cyclotron frequency. We chose $1000 \mathrm{~Hz}$ below the cyclotron frequency to avoid interference with any fragmentation processes. After excitation, the ions are accelerated until the phase lag between their coherent motion and the drive frequency equals $180^{\circ}$, and then they are decelerated to zero kinetic energy. This cycle is repeated as long as the irradiating field is applied accordingly. SORI-CID is a multiple collision process and provides high fragmentation efficiencies for large molecules.

The high-resolution and collision-dissociation FT techniques described above were used in this work to separate and identify two by-products of the polymerization between 12-hydroxystearic acid (HSA) and stearic acid (SA), to provide precise information about the exact location of caprolactones on the Tris(2-hydroxyethyl)isocyanurate (THEIC) molecule, and to sequence a GMA/MMA copolymer.

\section{Experimental}

Materials Potassium iodide and sodium iodide were purchased from Sigma-Aldrich (St. Louis, MO, USA). HPLCgrade tetrahydrofuran (THF) and methanol were obtained from JT Baker (Phillipsburg, NJ, USA). The polymerization of the HSA [24], the preparation of the caprolactone oligomer adduct [25], and the preparation of the GMA/ MMA copolymer[26] are reported and described elsewhere.

Mass spectrometry The direct electrospray ionization experiments were conducted in a commercial Analytica ESI source coupled to a Finnigan 3 Tesla Fourier transform mass spectrometer (Madison, WI, USA) with a dual-cell configuration. The polymer samples were dissolved in tetrahydrofuran (THF) to a concentration of $10^{-4} \mathrm{M}$, and were introduced into the gas phase through a three-layer needle. The cationization agent, $\mathrm{NaI}$, was dissolved in methanol at $10^{-3} \mathrm{M}$, and introduced through a sheath flow.

Laser desorption experiments were performed using a Tachisto $\mathrm{CO}_{2}$ laser (Needham, MA, USA) $(\lambda=10.6 \mu \mathrm{m}$, power $=10^{6}-10^{8} \mathrm{~W} / \mathrm{cm}^{2}$, pulse width $=40-80 \mathrm{~ns}$ ) on the FTMS instrument. The detailed experimental procedure is published elsewhere [8]. In brief, the polymer samples $(100 \mu \mathrm{L}$ of $10 \% \mathrm{w} / \mathrm{v}$ in THF) were deposited onto a cellulose substrate disc. A cationization agent $(50 \mu \mathrm{L}$ of a saturated solution of KI in methanol) was added to the cellulose substrate disc on top of the polymer samples. The disc, along with sample and cationization agent, was placed in an oven for $15 \mathrm{~min}$ to remove the solvent before being introduced into a vacuum chamber through a Finnigan commercial Autoprobe assembly. Ions formed by laser desorption were trapped in the source cell, excited, and detected. 
Sustained off-resonance irradiation collision-induced dissociation (SORI-CID) was performed in a 3-T FTMS instrument. Ions generated from the ESI source were transferred into the ICR (ion cyclotron resonance) cell through ion transfer lenses. The ions of interest were then isolated using SWIFT (stored waveform inverse Fourier transform) and excited by applying RF (radiofrequency) power at $700-1000 \mathrm{~Hz}$ below their cyclotron frequency, followed by the introduction of a gas pulse of Ar. Ions with adequate kinetic energy will collide with $\mathrm{Ar}$ gas and fragment. The parent ions and resulting fragment ions were subsequently trapped, excited and detected in the ICR cell.

\section{Results and discussion}

Separation and detection of the polymerization products of 12-hydroxystearic acid (HSA) and stearic acid (SA)

Polyesters are common components of automotive coatings, where they are often used as dispersants to prevent pigment aggregation and maintain sprayable viscosities. Mass spectrometry was applied for the structural characterization of the early stages of a comb dispersant synthesis, since the performance of a coating is strongly related to the structural features of its polyester building blocks.

To achieve the detailed structural characterization of polymer end-group components, direct laser desorption (LD) performed on a FT mass spectrometer is a quick and relatively simple method of analysis, since it does not require a compatible matrix or lengthy sample preparation procedures. The drawback of this technique is the poor resolution due to the high pressure introduced by the laser pulse. For ultrahigh resolution with LD, quadrupolar excitation activation (QEA) techniques were applied in this work to determine the end-group compositions of 12hydroxystearic acid (HSA) and stearic acid (SA) polyesters. This work presents the mass spectrometry characterization and discussion of only the first step of this synthesis; the final graft polymer was beyond the upper mass capability of the instrument.

As shown in Scheme 1, linear HSA oligomers are synthesized through the solid-state condensation polymerization of HSA and SA monomers in a molar ratio of six to one.

HSA oligomers are then reacted with the epoxy group of glycidyl methacrylate (GMA) to form functionized oligomers that possess a terminal double bond. The double bond can be subsequently free-radically polymerized with acrylates to form graft polymers. These types of polymers are utilized as dispersants, adhesives and compatibilizers in the coatings industry. As illustrated in Scheme 1, two by-products, cyclic HSA and SA-capped HSA, are also formed during the first step of the synthesis, with longer reaction times favoring cycle formation. Since the cyclic by-product has no functional group for further crosslinking, it is essential to determine its presence and amount in the reaction mixture. It is not certain that the cyclized material is not a linear isomer with a double bond. However, when reacted with glycidyl methacrylate in step two of Scheme 1, the material that we termed "cyclized" did not react. Therefore, we concluded that the HSA forms a cyclic structure.

Figure 1a shows the LD/FTMS spectrum of the HSA oligomer reaction products. The distribution extends from
Scheme 1 Synthesis and functionalization of HSA oligomers and graft polymers. $S A$, stearic acid; HSA, 12-hydroxystearic acid; GMA, glycidyl methacrylate

Synthesis of 12-Hydroxystearic Acid Oligomers:

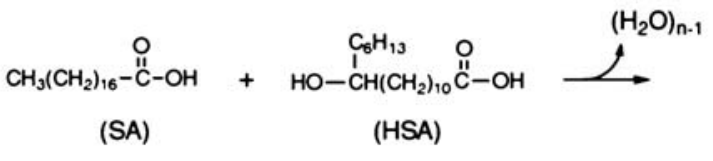

Fuctionalization of HSA Oligomers (Epoxy/Acid Reaction):

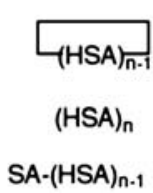

Graft Polymer Synthesis:

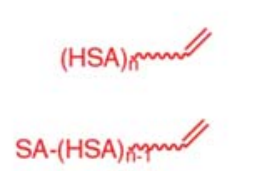

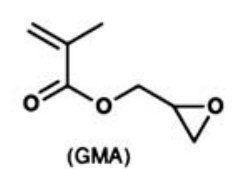

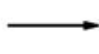

(GMA)

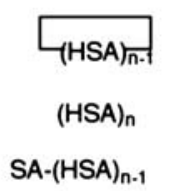

SA-(HSA) $n-1$ 
Fig. 1 LD/FTMS of HSA oligomers. a Full LD/FTMS spectrum. b LD/FTMS of the pentamer. c High-resolution spectrum of the pentamer achieved with QEA. $R P$, resolving power

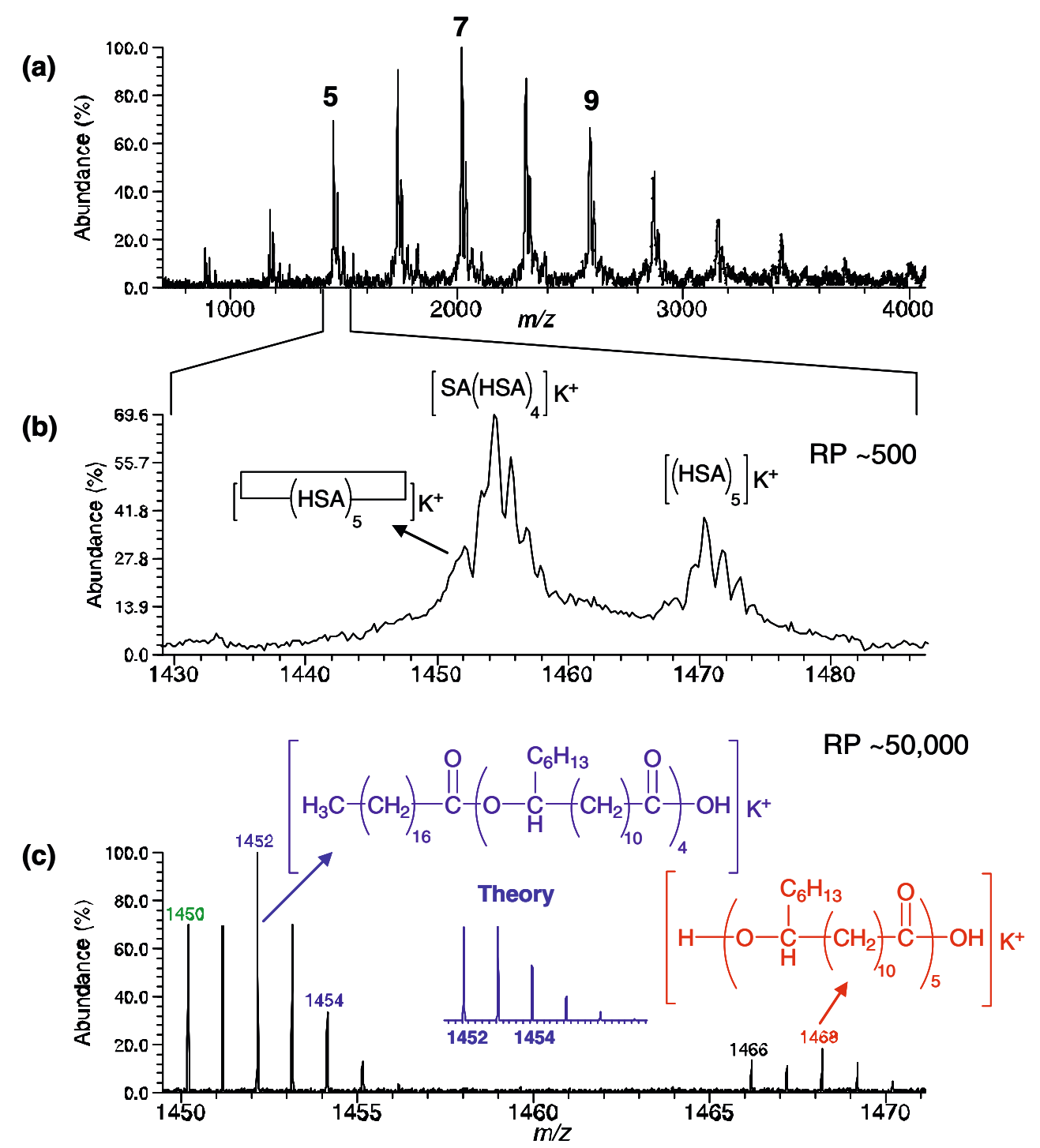

$m / z 900$ to 3,600 , with a maximum mass distribution at approximately $\mathrm{m} / \mathrm{z} 2,000$, corresponding to about seven units of of HSA. The spacing between the major peaks is $\mathrm{m} / \mathrm{z} 282$, which corresponds to the mass of the repeat unit of HSA, 300 Daltons (Da) minus $18 \mathrm{Da}=282 \mathrm{Da}$.

Figure $1 \mathrm{~b}$ is an expansion of the pentamer region. The three peaks labeled in the mass spectrum correspond to three possible product ions (pentamer cyclic HSA, tetramer SA-capped HSA, and pentamer linear HSA oligomers) with $\mathrm{K}^{+}$attached. It is clear that the mass resolution of the LD spectrum is not sufficient to separate the pentamer cyclic HSA and tetramer SA-capped HSA ions, since their masses differ by only $m / z 2$. However, the ultrahigh resolution achieved by the QEA experiments allowed the separation and accurate determination of the two species.

QEA was performed over a narrow mass range, extending from $\mathrm{m} / z 1,450$ through 1,470 . The two ions of interest are separated with mass resolution of up to 50,000, as shown in Fig. 1c. They correspond to the oligomers of the pentamer cyclic HSA $(\mathrm{m} / z$ 1,450, shown in green) and SAcapped tetramer linear HSA $(\mathrm{m} / \mathrm{z} 1,452$, shown in blue). The data confirm that both compounds are present, with cyclic HSA being the more abundant product, an invaluable piece of information to a coatings formulator which can only be obtained with the ultrahigh resolution offered by QEA FTMS. The theoretical isotopic distribution does not match the experimentally determined isotopic ratio due to the overlap with the isotopic contribution from the cyclized product.

This example shows the powerful problem-solving capability of high-resolution mass spectrometry and its importance in coatings characterization. However, even though it is unique in terms of the high resolution it offers, FT alone cannot address all of the challenges posed by polymer and 
Scheme 2 Isomeric structures of the $4 / 1$ product of the THEIC and caprolactone reaction. a Desired structures (A type). b Unwanted structures (B type). $H y$, hydroxyethyl; $C L$, caprolactone<smiles></smiles><smiles>O=c1n(Cl)c(=O)n(C(Cl)(Cl)Cl)c(=O)n1CCl</smiles>

A-type structures

(b)<smiles>O=c1[nH]c(=O)n(CCl)c(=O)n1[AlH2]</smiles><smiles>O=c1n([AlH2])c(=O)n([Hg]Cl)c(=O)n1[AlH2]</smiles>

\section{B-type structures}<smiles>O=c1n(Cl)c(=O)n(CCl)c(=O)n1CCl</smiles><smiles>O=c1n([Hg])c(=O)n(CCl)c(=O)n1CCl</smiles>

coatings characterization. In such cases, the coupling of FT with tandem mass spectrometry has proven to be an excellent tool for analyses.

Detailed structural elucidation of Tris(2-hydroxyethyl) isocyanurate (THEIC)/caprolactone adducts

A new coating composition, invented for better mar resistance when applied to an automotive surface, consists of acrylic polymers, melamine, and Tris(2-hydroxyethyl) isocyanurate (THEIC)) extended with an aliphatic caprolactone oligomer [27]. An improved mar resistance can provide an improved overall appearance by increasing the clarity of the topcoat and its resistance to deterioration. The coating composition can be formulated at $100 \%$ solids. However, the films have a poor appearance (haze), which may be attributed to the presence of poly(caprolactone) homopolymer. There is a need for coating formulations that provide a better balance of performance characteristics after application; mar-resistant coatings were attained by softening the coating, which can unfortunately reduce other performance characteristics.

The reaction product of THEIC and caprolactone can form two different types of structures. The desired structure has a caprolactone molecule attached to each of the three hydroxyethyl moieties. In this case, the probabilities of the caprolactone molecules reacting with the hydroxyethyl groups or with themselves are the same. Scheme 2a shows the formation of the desired structures (A type) of the 4/1 product of THEIC and caprolactone (four caprolactone units and one THEIC). None of the three isomeric structures shown in Scheme 2a has a free hydroxyethyl group. Scheme $2 b$, on the other hand, shows the formation of the unwanted B-type structures, where some of the hydroxyethyl groups have not reacted with caprolactone. In this case, the molecules contain long chains of poly (caprolactone) homopolymer along with hydroxyethyl groups. The A- and B-type structures are isomeric and have the same molecular weight. Since single-stage MS is not able to distinguish them, MS/MS experiments become necessary to isolate the isomeric molecular ions and fragment them. Structural information can be obtained from the differences in fragmentation patterns.

Figure 2a shows the ESI mass spectrum of THEIC/ caprolactone. A distribution with a repeating mass of $\mathrm{m} / \mathrm{z}$ 114 due to the caprolactone $\left(\mathrm{C}_{6} \mathrm{H}_{10} \mathrm{O}_{2}\right)$ is observed. The ESI spectrum shows that up to nine caprolactones are added to THEIC with the most abundant peak at $\mathrm{m} / \mathrm{z} 740$, the addition of four caprolactones. However, the data could not provide precise information about how caprolactones were added to THEIC and whether it exhibits A- or B-type structures.

Figure 2c shows the MS/MS spectrum of the most abundant ion at $\mathrm{m} / \mathrm{z} 740$. The two dominant peaks at $\mathrm{m} / \mathrm{z}$ 383 and 497 correspond to three caprolactone and four sequential homo-caprolactone molecules, respectively. The spectrum represents a molecule that contains long runs of caprolactone chains, which is indicative of a B-type structure. This interpretation is supported by ${ }^{13} \mathrm{C}$-NMR data, which show at least two types of hydroxyl groups for THEIC/caprolactone. The MS/MS results confirm that the compound contains long segments of caprolactone that could result in a poor, hazy appearance of the coating films.

\section{GMA/MMA sequencing}

It has been reported that functional monomers such as glycidyl methacrylate (GMA) can be efficiently converted to low molecular weight (degree of polymerization "dp" 3 ) oligomers [28]. These dimers and trimers are used as chaintransfer agents with other monomers to ensure functionality on every chain [26]. Moreover, the data show that the resulting polymers have the functional monomer located at 
Fig. $2 \mathrm{MS} / \mathrm{MS}$ of the THEIC

caprolactone oligomer adduct. a

ESI of the THEIC caprolactone

adduct. b Isolation of

$\left[\mathrm{CL}_{4}\right.$ THEIC $] \mathrm{Na}^{+}$. $\mathbf{c}$ SORI of

$\left[\mathrm{CL}_{4}\right.$ THEIC $] \mathrm{Na}^{+}$. CL,

caprolactone (a)

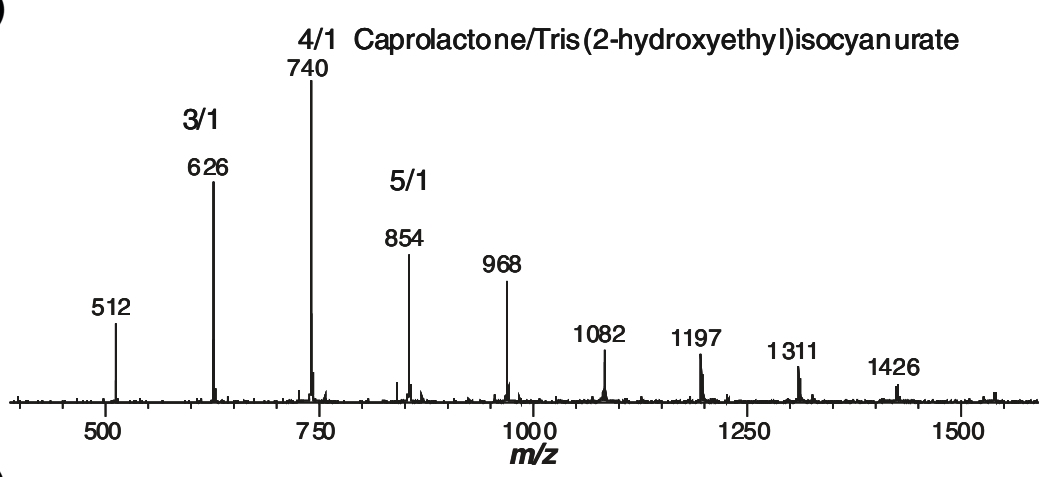

(b)

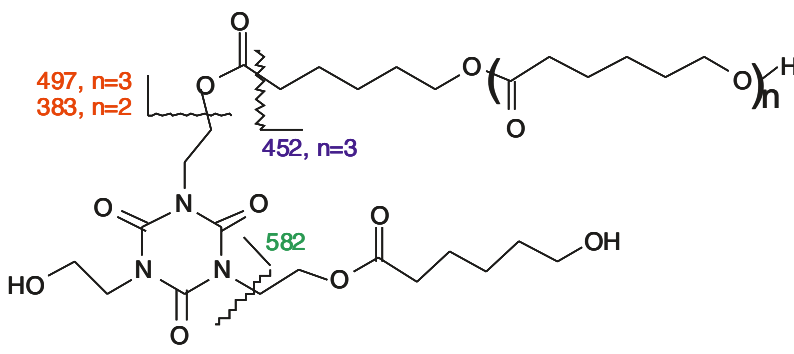

(c)
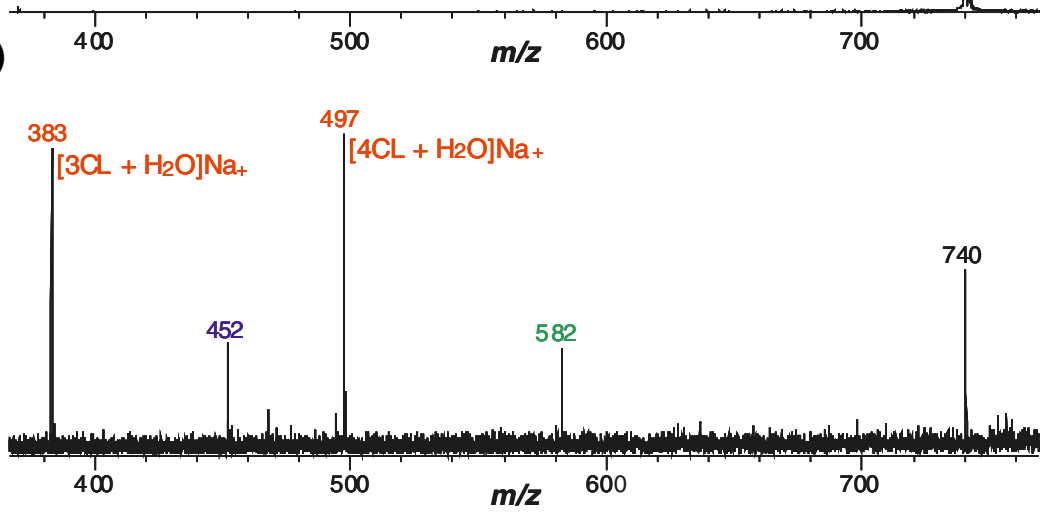

the chain termini (telechelics), where it is thought to be the most effective for crosslinking.

Model telechelics were prepared using glycidyl methacrylate (GMA) dimer and methylmethacrylate (MMA) monomer. All polymer chains were expected to possess a sequence of MMAs initiated by a GMA and terminated by a GMA possessing a vinyl group, as depicted in Scheme 3. Owing to the success of our previous work on acrylic copolymers, we chose electrospray ionization as the preferred method of soft ionization [6].
Scheme 3 Macromonomer approach to telechelics. GMA/ MMA telechelic synthesis via macromonomers, producing a functionality of at least two per chain

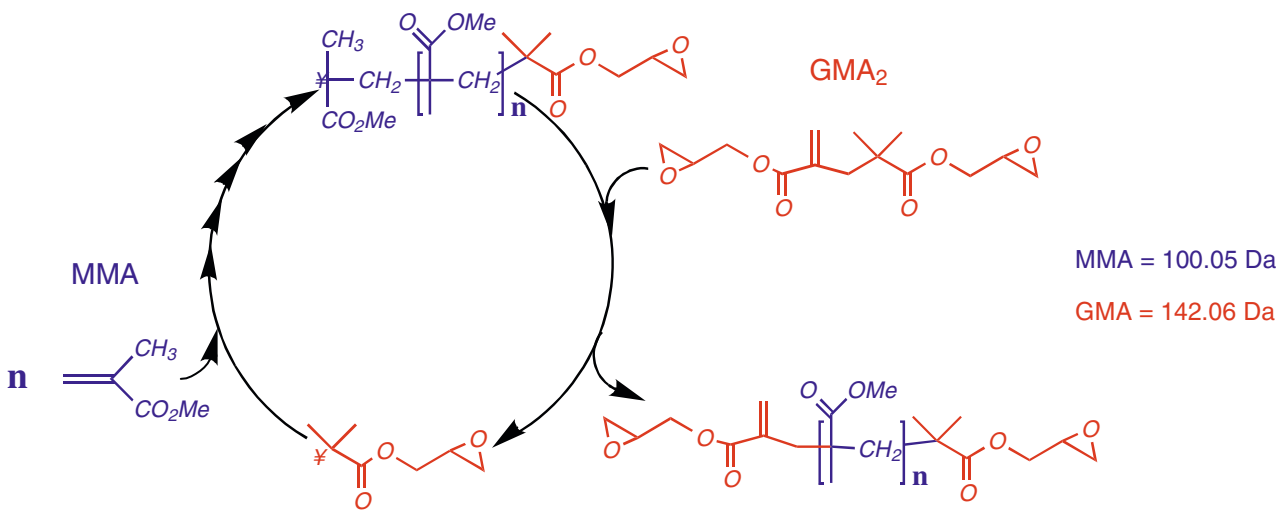


Fig. 3 SORI-CID of sodiated GMA pentamer

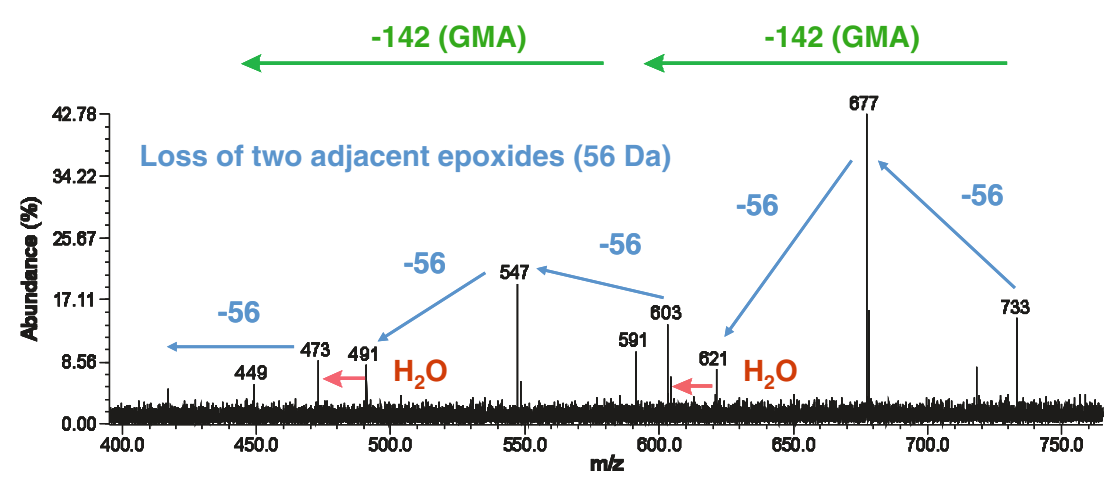

NMR analysis revealed that one GMA was terminal with a vinyl group. The remaining question was the location of the other GMA in the GMA/MMA copolymer; the second GMA monomer could be adjacent to the terminal GMA or located at the other end of the chain. To answer this question, SORI-CID was performed on a model homopolymer, the sodiated GMA pentamer at $m / z$ 733, to determine the fragmentation pattern of adjacent GMAs. Should a similar fragmentation pattern be observed in the GMA/MMA copolymer, the GMA units can be considered adjacent. If no similarities are detected between the fragmentation of the GMA homopolymer and the GMA/MMA copolymer, both GMA units in the copolymer can be considered terminal. Figure 3 displays the spectrum of the GMA pentamer.

Little backbone cleavage was observed, as shown by the loss of $\mathrm{m} / \mathrm{z} 142$ (the weight of GMA) between $\mathrm{m} / \mathrm{z} 591$ and (a)

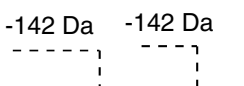<smiles>C=C(CC(C)(CC(C)(CC(CI)(CC(C)(C)C(=O)OCC1CO1)C(=O)OCC1CO1)C(=O)OCC1CO1)C(=O)OCC1CO1)C(=O)OCC1CO1</smiles>
Loss of two adjacent epoxides (56 Da) with $\mathrm{H}$-transfer<smiles>C=C(CC(C)(CC(C)(CC)C(=O)OCC1CO1)C(=O)OCC1CO1)C(=O)OCC1CO1</smiles><smiles>C=C(CC(C)(CC(C)(CC1(C)CC(C)(C)C(=O)OC1=O)C(=O)OCC1CO1)C(=O)OCC1CO1)C(=O)OCC1CO1</smiles>

(b)

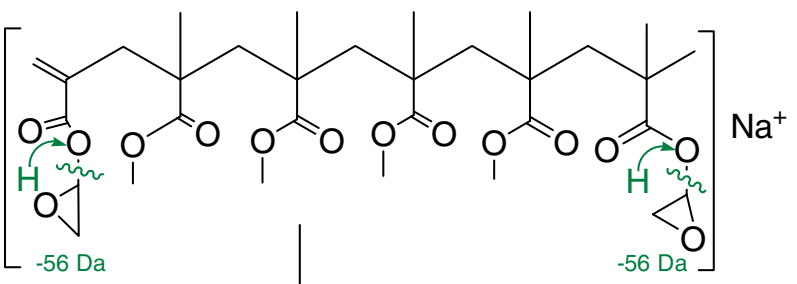<smiles>COC(=O)CC(C)(C)CC(C)(CC(C)(CC(C)(CC(C)(C(=O)OC)C(=O)OC)C(=O)OC)C(=O)OC)C(=O)OC</smiles><smiles>C=C1CC(C)(CC(C)(CC(C)(CC2(C)CC(C)(C[NH3+])C(=O)OC2=O)C(=O)OC)C(=O)OC)C(=O)OC1=O</smiles>

Scheme 4 Fragmentation pattern in SORI-CID. a GMA pentamer. b GMA/MMA copolymer 
449. However, two losses of glycidyl $(\mathrm{m} / \mathrm{z}=677,621)$ were observed, followed by the loss of water $(m / z=603)$ to form anhydride. Furthermore, the anhydride loses additional glycidyl groups $(\mathrm{m} / \mathrm{z} 547,491)$, followed by another dehydration (Scheme 4a). Thus, the SORI-CID behavior of adjacent GMAs is consecutive losses of $m / z 56$ followed by dehydration $(\mathrm{m} / \mathrm{z} 18)$.

Figure 4 displays the spectrum of the GMA/MMA copolymer obtained using $\mathrm{CO}_{2}$ laser desorption FTMS with potassium cation doping; the GMA monomer has a mass of $142 \mathrm{Da}$, whereas the MMA monomer has a mass of 100 Da. Laser desorption FTMS analysis shows that over $90 \%$ of the oligomeric chains contain two GMAs with a sequence of MMA, whereas the other $10 \%$ correspond to chains containing one GMA unit only (Fig. 4a). Using axialization and collisional cooling, it was also shown that all of the chains were terminated with a double bond, thus supporting the mechanism shown in Scheme 4.
The polymer composition was confirmed by acquiring the high-resolution spectrum. Figure $4 \mathrm{~b}$ shows the ultrahigh resolution mass spectrum of 13-mer to 17-mer obtained by QEA experiments. The experimental data is consistent with the theoretical isotope distribution, as shown in Fig. $4 \mathrm{~b}$ in the inset.

Sustained off-resonance irradiation collision-induced dissociation (SORI-CID) was performed on electrosprayed sodiated polymer ions to determine the exact locations of the GMAs. Figure $4 \mathrm{c}$ displays the SORI-CID spectrum of the $\left[\mathrm{GMA}_{2} / \mathrm{MMA}_{4}\right] \mathrm{Na}^{+}$. No backbone cleavage was observed, but consecutive losses of $\mathrm{m} / \mathrm{z} 56$ are attributed to loss of glycidol, which were followed by losses of methanol, $\mathrm{m} / \mathrm{z}$ 32). This fragmentation pattern corresponds to two GMA units with terminal locations in the $\left[\mathrm{GMA}_{2} /\right.$ $\mathrm{MMA}_{4}$ ] chain (Scheme $4 \mathrm{~b}$ ), rather than those adjacent to each other. If the latter case were true, the SORI-CID results would display a loss of water, as observed in the
Fig. 4 FTMS of GMA/MMA telechelic polymer. a LD/FTMS spectrum. b High-resolution (QEA) FTMS spectrum of 13mer to 17-mer. c ESI SORI-CID of $\left[\mathrm{GMA}_{2} / \mathrm{MMA}_{4}\right] \mathrm{Na}^{+}$

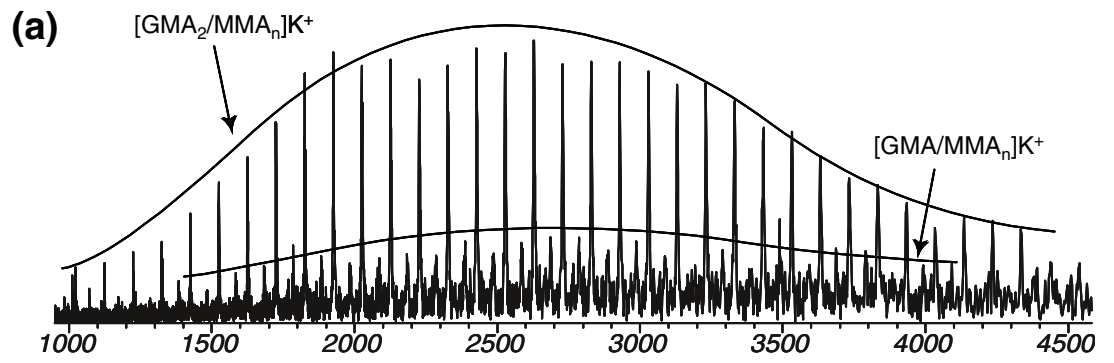

(b)

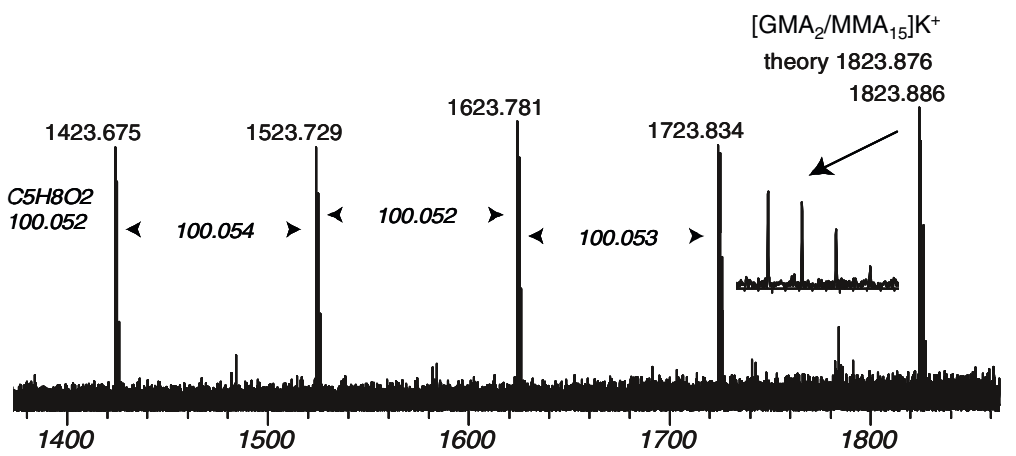

(c)

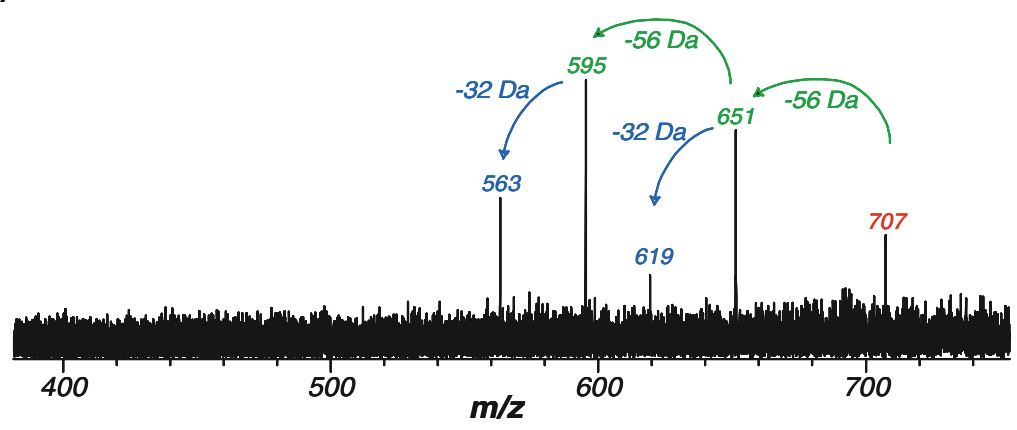


spectrum of the GMA homopolymer (Fig. 3). Instead, the GMA/MMA SORI-CID spectrum shows two losses of methanol, which is indicative of a telechelic copolymer composition. NMR (heteronuclear multiple bond correlation through the double bond) confirms one GMA per chain terminus. A statistical distribution of GMAs, not just two per chain, is expected unless the remaining GMA is specifically located at the other chain terminus. The data support a telechelic copolymer.

\section{Conclusions}

Advanced mass spectrometry provides extensive information about high-solids automotive coatings, such as accurate molecular weight distributions, end-group analysis, and structural elucidation. Unwanted by-products that are known to cause manufacturing problems and are expected to impact negatively on the long-term durability were accurately identified by MS/MS. In addition, MS/MS experiments were conducted to obtain sequence information on the copolymers and oligomeric additives, whereas ultrahigh mass resolution was performed to separate molecules that are close in mass.

Acknowledgments The authors thank R. Celikay for performing the experiments. B.P. Devlin, C.T. Berge, S. K. Quashie and P.H. Corcoran synthesized the organic compounds characterized in this study. The authors are grateful to DuPont Performance Coatings for their permission to publish this investigation.

\section{References}

1. Adamsons K, Blackman G, Gregorovich B, Li L, Matheson R (1998) Prog Org Coat 34:64-74

2. Dole M, Mack LL, Hines RL, Mobley RC, Ferguson LD, Alice MB (1968) J Chem Phys 49:2240
3. Fenn JB, Mann M, Meng CK, Whitehouse CM (1989) Science 246:64

4. Fenn JB (1993) J Am Soc Mass Spectrom 4:524

5. Marshall AG, Grosshans PB (1991) Anal Chem 63:215

6. Shi SDH, Hendrickson CL, Marshall AG, Simonsick WJ, Aaserud DJ (1998) Anal Chem 70:3220-3226

7. Brown RS, Weil DA, Wilkins CL (1986) Macromolecules 19:1255-1260

8. Simonsick WJ Jr, Ross CW III (1996) Int J Mass Spectrom Ion Proc 157:379

9. Hanton SD (2001) Chem Rev 101:527-569

10. Jackson AT, Jennings KR, Scrivens JH (1997) J Am Soc Mass Spectrom 8:76-85

11. Jackson AT, Yates HT, Scrivens JH, Green MR, Bateman RH (1997) J Am Soc Mass Spectrom 8:1206-1213

12. Chen R, Tseng AM, Uhing M, Li L (2001) J Am Soc Mass Spectrom 12:55-60

13. Lattimer RP, Munster H, Budzikiewicz H (1989) Int J Mass Spectrom Ion Proc 90:119-129

14. Lattimer RP (1992) J Am Soc Mass Spectrom 3:225-234

15. Rizzarelli P, Puglisi C, Montaudo G (2006) Rapid Commun Mass Spectrom 20:1683-1694

16. Jackson AT, Williams JP, Scrivens JH (2006) Rapid Commun Mass Spectrom 20:2717-2727

17. Jackson AT, Slade SE, Scrivens JH (2004) Int J Mass Spectrom 283:265-277

18. Pastor SJ, Castoro JA, Wilkins CL (1995) Anal Chem 67:379

19. Arnould MA, Vargas R, Buehner RW, Wesdemiotis C (2005) Eur J Mass Spectrom 11:243-256

20. Schweikhard L, Guan S, Marshall AG (1992) Int J Mass Spectrom Ion Proc 120:71

21. Guan S, Kim HS, Marshall AG, Wahl TD, Wood XX (1994) Chem Rev 94:2161

22. Solouki T, Reinhold BB, Costello CE, O’Malley M, Guan S, Marshall AG (1998) Anal Chem 70:857-864

23. Pastor SJ, Wilkins CL (1998) Int J Mass Spectrom Ion Proc 175:81-92

24. Simms JA, West MWJ (1995) US Patent 5,424,364

25. Corcoran PH, Hazan I, Quashie SK (1998) US Patent 5,447,590

26. Delvin BP, Darling TR, Berge CT, Darmon MJ, Grady MC, Hansen JE, Simonsick WJ, Matheson RR, Litty LL, Paquet DA, Wilczek L, Gridnev A (1997) Polym Prepr 38:458

27. Corcoran PH, Hazan I, Quashie SK (2008) US Patent 5,447,590

28. Janowicz AH, Melby LR (1987) US Patent 4,680,352 